\title{
Foreword: Special issue on particle methods for the oil and gas industry
}

\author{
Christopher R. Leonardi ${ }^{1,2}$
}

Published online: 9 March 2016

(C) OWZ 2016

It is with great pleasure that I introduce this Special Issue of Computational Particle Mechanics, titled Particle Methods for the Oil and Gas Industry. It brings together 11 original research articles from academics around the world, all focused on an industry of international significance.

The landscape of global hydrocarbon production has undergone significant transformation in recent years. The emergence of unconventional sources of oil and gas, such as shales, tar sands and coal seams, has presented industry and government with myriad new problems related to sustainable and profitable production. Most notable of these is the reservoir stimulation process known as hydraulic fracturing, and the challenges associated with increasing its efficacy and repeatability whilst minimising its impact on the surrounding environment. This and other challenges present vast opportunities for research, which was reinforced by a recent report by the Chief Scientist and Engineer of New South Wales, Australia, that recommends that "the Government (should) continue and extend its role as a champion of research relevant to the hard problems related to under-earth especially the development of sophisticated predictive underground models." Further, both the United States Department

Christopher R. Leonardi

c.leonardi@uq.edu.au; chrisleo@mit.edu

1 School of Mechanical and Mining Engineering, The University of Queensland, Cooper Rd, St Lucia, QLD 4072, Australia

2 Department of Civil and Environmental Engineering, Massachusetts Institute of Technology, 77 Massachusetts Avenue, Cambridge, MA 02139, USA of Energy and the Massachusetts Institute of Technology, in recent reports on the production of natural gas, have championed for the development of models which can provide insight on the processes relevant to unconventional gas production.

Oil and gas production is of course a multidisciplinary industry and this aligns well with the numerous disciplines of science and engineering in which particle-based methods are now routinely applied. The diversity of the work collated in this issue, both in terms of numerical methods and industrial contexts, is a testament to that. One of the aspects of particle-based methods that contributes to this wide range of applicability is the way in which they can be interpreted. For example, the discrete element method may be used to model an assemblage of distinct particles (e.g. the flow of a pile of sand), discretise a fundamentally granular continuum (e.g. the loading of a sandstone specimen), or perform both at the same time (e.g. continuum-to-discrete fracture). The generality of the fundamental kernel also facilitates the traversing of scales, as the change in interaction forces (along with some other details!) allows the evolution from molecular dynamics at the microscale, to dissipative particle dynamics at the mesoscale, and the discrete element method and smoothed particle hydrodynamics at the macroscale. Lastly, the ability of particle-based methods to couple with other numerical methods such as the finite element method and the lattice Boltzmann method (a particle-based method in its own way) opens research avenues in the areas of fluid-structure interaction, suspension transport, and more.

The editorial review of works related to drilling, hydraulic fracturing, porous media flows, and marine structures has been both stimulating and rewarding. I would like to take this opportunity to thank the many authors, both new to Computational Particle Mechanics and those returning, for their contributions to this issue. I would also like to thank the 
reviewers, many of whom have been introduced to the journal through this process, for their time and valuable critical insight. Finally, I would like to acknowledge the Editors-in-
Chief, Prof. Tarek I. Zohdi, Prof. Eugenio Oñate, and Prof. Peter Wriggers, for providing me with the opportunity to curate this issue. It has been fun. 